\title{
Hyperemesis gravidarum and gestational transient hyperthyroidism: A case report
}

\author{
Cheau Wei, Chin*, Aye Aye Myint \\ Jeffrey Cheah School of Medicine and Health Sciences, Clinical School Johor Bahru, Bukit Azha, Johor Bahru, Malaysia
}

Email address:

cheauwei.1808@gmail.com (Cheau Wei, Chin), a2myintster@gmail.com (A. A. Myint)

\section{To cite this article:}

Cheau Wei, Chin, Aye Aye Myint. Hyperemesis Gravidarum and Gestational Transient Hyperthyroidism: A Case Report. Journal of Gynecology and Obstetrics. Vol. 1, No. 2, 2013, pp. 7-10. doi: 10.11648/j.jgo.20130102.11

\begin{abstract}
Gestational transient hyperthyroidism is often associated with hyperemesis gravidarum, which is a relatively uncommon condition in women during the first and second trimester of pregnancy. It is a transient phenomenon which resolves itself by the 20th gestational week. This is a report of a case of a pregnant woman with hyperemesis gravidarum and gestational transient hyperthyroidism who was admitted to a government hospital for 1 month.
\end{abstract}

Keywords: Hyperemesis Gravidarum, Gestational Transient Hyperthyroidism, Pregnancy

\section{Introduction}

Hyperemesis gravidarum (which occurs in $0.3-1 \%$ of pregnancies) ${ }^{1}$ and gestational transient hyperthyroidism (which occurs in $1-2 \%$ of pregnancies) ${ }^{3}$ are associated with elevated hCG levels during pregnancy ${ }^{2}$. It is important to distinguish gestational transient hyperthyroidism from Graves' disease because the course, fetal outcomes, management, and follow-up are different ${ }^{3}$. Graves' disease should be suspected if there is presence of goitre ${ }^{1}$ and/or persistent abnormal thyroid function test result after 20th gestational week ${ }^{5}$. Gestational transient hyperthyroidism usually resolveed by then, when hCG levels decline ${ }^{3}$.

The aim of this case report is to highlight the clinical presentation and transient phenomenon of gestational transient hyperthyroidism with hyperemesis gravidarum, and also to emphasize on the recommended management.

\section{Case Report}

A 30 year-old pregnant Indian lady, $14^{\text {th }}$ week of pregnancy (Gravida 2, Para 1) was admitted to a hospital in Johor with frequent vomiting of more than 15 times daily, epigastric pain since her $10^{\text {th }}$ week of pregnancy. The vomitus was mainly water and saliva as she was unable to tolerate orally, but she had two episodes of hematemesis in mid of April. She also complains of nausea, dizziness, lethargy, loss of appetite and weight, constipation, and right-sided headache. She denies symptoms suggestive of hyperthyroidism, such as fever, palpitation, agitation, diarrhea, and heat intolerance. She underwent caesarean section when giving birth to her first child due to breech birth. However she did not have hyperemesis gravidarum in her first pregnancy. She has no known medical illnesses and non-remarkable family history of thyroid disease.

Upon physical examination, the patient was afebrile, alert and conscious but dehydrated. Her pulse rate was 84 beats per minute with blood pressure of $117 / 70 \mathrm{mmHg}$. No hand tremors were observed. There was no conjunctiva pallor, but the patient was jaundiced. In addition, there were no eye signs of Graves' opthalmopathy and palpable goiter. Her lungs were clear and normal heart sounds (S1, S2) were heard with no murmurs. Her abdomen was soft but tenderness at right hypochondrium and epigastric region. The rest of the examinations were unremarkable.

Her laboratory test results showed consistent normocytic normochromic anemia, elevated bilirubin, ALT and AST, but low serum creatinine, serum potassium, magnesium and creatine kinase. The thyroid function test also shows evidence of thyrotoxicosis (elevated T4 and low TSH level) during $14^{\text {th }}$ gestational week, but the values normalized during 15th gestational week as shown in Table I. However the TSH receptor antibodies, anitinuclear antibody test, antithyroglobulin antibody test and anitimicrosomal antibody test results show negative. Furthermore, the investigations for hepatitis $\mathrm{A}, \mathrm{B}, \mathrm{C}$ and the acid fast bacilli test, also show non-reactive. 
Table 1.Thyroid function test of patient

\begin{tabular}{ccccc}
\hline & $\begin{array}{c}\text { Reference } \\
\text { value }\end{array}$ & $\begin{array}{c}\text { 14th } \\
\text { gestational } \\
\text { week }\end{array}$ & $\begin{array}{c}\text { 15th } \\
\text { gestational } \\
\text { week }\end{array}$ & $\begin{array}{c}\text { 17th } \\
\text { gestational } \\
\text { week }\end{array}$ \\
\hline $\begin{array}{c}\text { Free T4 } \\
(\mathrm{pmol} / \mathrm{L}) \\
\mathrm{TSH}\end{array}$ & $10-20$ & 45.00 & 18.40 & 13.56 \\
$(\mathrm{miu} / \mathrm{L})$ & $0.2-4.0$ & 0.090 & 0.030 & 0.776 \\
\hline
\end{tabular}

The patient was given an intravenous drip of dextrose saline with potassium chloride throughout her stay in the hospital. Antithyroid medication was not introduced though her thyroid function showed hyperthyroidism. Her thyroid function test results normalize itself during her $15^{\text {th }}$ gestational week. Her frequent vomiting was significantly reduced and was able to tolerate orally. Her general condition was stable and she was discharged after 4 weeks of hospital admission.

\section{Discussion}

\subsection{Incidence and Prevalence}

Hyperemesis gravidarum is defined as intractable excessive vomiting during pregnancy with onset before the 13th gestational week, usually the woman is unable to tolerate orally and requires intravenous hydration ${ }^{1}$. It is likely to be associated with hyperthyroidism secondary to the high hCG level. Various studies showed $0.3-1 \%$ prevalence of $\mathrm{HG}$ in pregnancy, with a mean onset during the 3 rd gestational week, peaks in $11^{\text {th }}-13^{\text {th }}$ gestational week $^{2}$, and subsides after $14^{\text {th }}-18^{\text {th }}$ gestational week $^{3}$. Hyperemesis gravidarum is possibly associated with signs of disturbed nutritional status (alterations in electrolyte balance, more than 5\% weight loss, ketosis, acetonuria), neurological disturbances, retinal hemorrhage, liver and renal damage ${ }^{2}$.

On the other hand, the most common thyroid disease in all pregnancies is Graves' disease $(85-90 \%)$, while the secondly most common is gestational transient hyperthyroidism, which its incidence is $1-2 \%$ in all pregnancies ${ }^{3,4}$. Gestational transient hyperthyroidism is defined as firstly diagnosed hyperthyroidism in early pregnancy, which resolves spontaneously by the early second trimester of pregnancy, without evidence of autoimmune thyroid disease and physical findings associated with Graves' disease ${ }^{4}$. Gestational transient hyperthyroidism occurs up to two-thirds of women with hyperemesis gravidarum ${ }^{1}$.

\subsection{Pathophysiology of Gestational Transient Hyperthyroidism}

The actual pathophysiology of gestational transient hyperthyroidism is still not completely understood, but it is strongly associated with human chorionic gonadotropin (hCG) level during pregnancy. In normal pregnancy, hCG is produced by the placenta in the first gestational week, its level peaks at $10^{\text {th }}$ gestational week, then decreases and reaches a plateau by $20^{\text {th }}$ gestational week ${ }^{3}$. HCG and TSH have identical $\alpha$ subunit, which may lead to cross reactivity between the increased level of hCG and TSH receptor, leading to stimulation of $\mathrm{T} 3$ and $\mathrm{T} 4$, in return causing negative pituitary feedback of TSH, resulting in high T3 and T4 level, but low TSH, especially in $8^{\text {th }}-14^{\text {th }}$ gestational week, when hCG level peaks during pregnancy ${ }^{5}$. Several clinical studies have reported high hCG level in women with hyperemesis gravidarum, and there are evidences showing that hCG level is positively correlated with the severity of vomiting and the degree of thyroid stimulation ${ }^{1}$. In addition, studies also showed that serum hCG is inversely proportionate to serum $\mathrm{TSH}^{4}$. A study showed that hCG level in women with gestational transient hyperthyroidism remained abnormally elevated for weeks during the second trimester and free T4 levels normalizes parallel with the decline of hCG level, thus supporting the role of hCG in the pathogenesis of gestational transient hyperthyroidism $^{1}$, as shown in Figure 1.Figure 1 shows the relationship between serum hCG and $\mathrm{TSH}^{4}$.

Another explanation for gestational transient hyperthyroidism is that high estrogen level during pregnancy increases hepatic thyroid-binding globulin (TBG) synthesis, which reaches plateau during mid-gestation. The increased TBG level stimulates elevation of total T3 and T4 levels. The serum T4 level increases sharply between $6^{\text {th }}-12^{\text {th }}$ gestational week, and stabilizes around mid-gestation ${ }^{5}$.

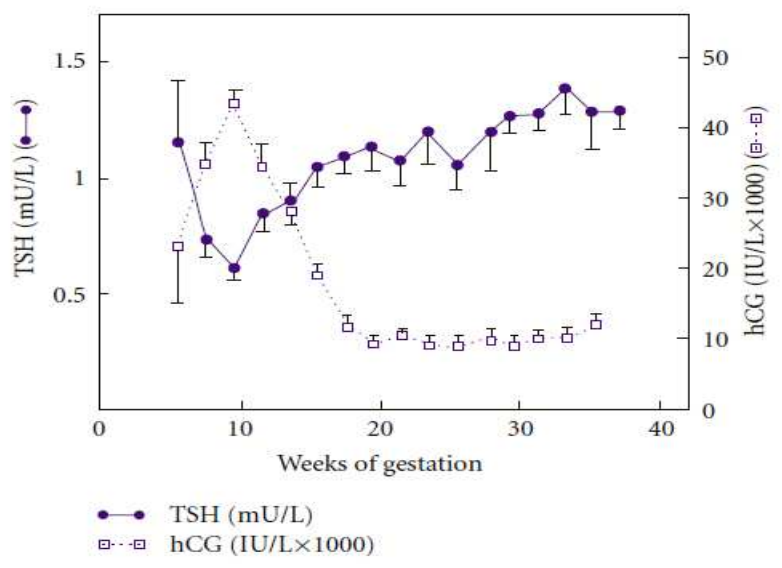

Figure 1. Relationship between serum $h C G$ and $T S H^{4}$

\subsection{Clinical Features}

Patients with hyperemesis gravidarum complicated with gestational transient hyperthyroidism usually complaint of nausea, vomiting and weight loss by gestational week 4-9, and present with tachycardia (secondary to dehydration), fine tremors and mild proximal weaknesses. In patients with high serum T3, they might present with shortness of breath, heat intolerance and palpitations. However, signs and symptoms suggestive of Grave's disease are absent ${ }^{5,6}$. A study showed that biochemical investigations in the early 
presentation of women with gestational transient hyperthyroidism and hyperemesis gravidarum reveal hyponatremia, hypokalemia, mild hyperbilirubinemia, and mild to moderate elevation of aspartate aminotransferase (AST) and/or alanine aminotransferase (ALT) levels. However, viral hepatitis screening shows negative results among those with abnormal liver function test results. The free T4 level is found to be elevated in the first trimester, but normalized by $15^{\text {th }}$ gestational week ${ }^{1}$ and serum TSH in the first trimester could be as low as $0.03-0.08 \mathrm{mIU} / \mathrm{L}$ secondary to the thyrotropic activity of $\mathrm{hCG}^{5}$.

Table 2.Clinical presentations and investigation results of patients with hyperemesis gravidarum complicated with gestational transient hyperthyroidism ${ }^{4}$

\begin{tabular}{|c|c|c|}
\hline Medical History & $\begin{array}{c}\text { Physical } \\
\text { Examination }\end{array}$ & Laboratory Tests \\
\hline - Absence of hyperthyroid symptoms prior & -No goiter & - Elevated free T4 \\
\hline conception & -Absence of Graves' ophthalmopathy or & - Suppressed or undetectable TSH \\
\hline - Similar history of vomiting in previous & dermopathy & - Negative thyroid antibodies: TPO and \\
\hline pregnancies & -No other physical findings such as vitiligo & TRAb, \\
\hline - Family history of Hyperemesis Gravidarum & and Plummer's nails & - Transient electrolyte abnormalities \\
\hline - No previous history of thyroid disease & -Signs of dehydration & - Abnormal liver function test results \\
\hline
\end{tabular}

\subsection{Management}

Women with hyperemesis gravidarum should be given supportive therapy with antiemetics, hydration, electrolyte replacement, and nutrition ${ }^{6}$. For those with hyperemesis gravidarum and gestational transient hyperthyroidism, anti-thyroid drugs (ATD) treatment is not recommended, as no benefit was supported by case reports and case studies. Gestational transient hyperthyroidism associated with hyperemesis gravidarum is of a transient nature where free T4 levels normalized by itself without ATD treatment ${ }^{1}$. Also, ATD treatment is poorly tolerated by patients, likely due to the persistent vomiting and metallic taste of $\mathrm{ATD}^{4}$. However, ATD treatment should be started if there is a persistence of hyperthyroid symptoms and thyroid function abnormalities after $18^{\text {th }}-20^{\text {th }}$ gestational weeks as this might indicate Graves' disease ${ }^{6,7}$.

\subsection{Nature Clinical Course of Gestational Transient Hyperthyroidism}

In women with hyperemesis gravidarum and gestational transient hyperthyroidism, the onset of nausea is usually within the 4 gestational weeks, worsens by the 9th gestational week and completely resolves by $20^{\text {th }}$ gestational week. The serum T4 is usually normalized by $15^{\text {th }}$ gestational week, but serum TSH may remain suppressed until the end of second trimester ${ }^{1,4}$. No significant obstetrical complications had been found among these women, but the infants born have lower birth weight compared to normal infants ${ }^{6}$.

\section{Conclusion}

In conclusion, gestational transient hyperthyroidism is a transient phenomenon which resolves itself by $20^{\text {th }}$ gestational week. Therefore, ATD treatment is not indicated unless the diagnosis is uncertain. Supportive management is the recommended treatment for hyperemesis gravidarum, and hospitalization may be required in severe cases. The main message of this case report is that the recommended management of patients with hyperemesis gravidarum complicated with gestational transient hyperthyroidism should be supportive management only and ATD treatment is unnecessary as the transient phenomenon of hyperthyroidism in pregnancy resolves itself.

\section{Author's Contribution}

This case report was completed in collaboration between both authors: Cheau Wei Chin gathered information regarding the patient, obtained consent from the patient and written the first and final draft of the case report, while Dr. Aye Aye Myint shared the literature reviews and advised on proofreading. Both authors have approved the final draft. We would also like to thank the Director General of Health Malaysia for permission to publish this paper.

\section{Consent}

Consent for publication has been obtained from the patient.

\section{Competing Interests}

Both authors have declared that no competing interest was present.

\section{References}

[1] Tan, Y.L, Loh, K.C., Yeo, S.H. et al. Transient hyperthyroidism of hyperemesis gravidarum. BJOG: an International Journal of Obstetrics and Gynaecology. June 2002; 109: 683-688.

[2] Nausea and vomiting in pregnancy. EBSCO DynaMed website. Available at: https://dynamed.ebscohost.com. Updated 10Apr 2013. Accessed June 20, 2013. 
[3] Goldman, A.M., and Mestman, J.H. Transient Non-Autoimmune Hyperthyroidism of Early Pregnancy. Journal of Thyroid Research. 2011; 2011: 142413.

[4] Jorge H. Mestman. Thyroid and Parathyroid Diseases in Pregnancy. Gabbe: Obstetrics: Normal and Problem Pregnancies, 6th ed. CHAPTER 40;929-933

[5] Yazbeck, C.F. and Sullivan, S.D. Thyroid Disorders during Pregnancy. Medical Clinics of North America 2012; 96:2, 235-256.
[6] Fitzpatrick D.L., and Russel M.A. Diagnosis and management of thyroid disease in pregnancy. Obstet Gynecol Clin North Am 2010; 37:173-93.

[7] CynthiaF. Yazbeck, MD, Shannon D. Sullivan, MD, PhD.Thyroid Disorders During pregnancy.Medical Clinics of North America - Volume 96, Issue 2 . 